\section{How, where and why(not) ... plant biotechnology clearly uncovered}

\author{
Plant Biotechnology: The Genetic \\ Manipulation of Plants \\ Edited by A Slater, N Scott and M Fowler \\ Oxford University Press, New York; 2003. 346 pp. \\ $£ 19.99$, paperback. ISBN 0-19-9254680.
}

Heredity (2004) 93, 114. doi:10.1038/sj.hdy.6800472

Reviewed by RS Conlan

The field of plant biotechnology is currently under intense scrutiny at all levels. While the great potential benefits achievable with this technology are now attainable due to scientific advances, the future of the technology is under much debate. 'Plant Biotechnology' offers a comprehensive and unbiased overview of both the technology and its applications, and also examines current perspectives in the field. The text aims to provide a solid grounding in plant biotechnology with particular reference to applications with crop plants where genetically modified varieties have been created and are today being farmed in several countries. Targeted at the advanced undergraduate and also suited as an introduction for postgraduates and nonexperts, 'Plant Biotechnology' is one of the best texts currently available in the field, and is a much needed addition to the literature.

After introducing plant genomes and their expression, detailed coverage is given to plant tissue culture and transformation including delivery vectors. This section, however, does not contain enough information on gene suppression via RNA (antisense and RNAi), which is covered elsewhere, but in insufficient detail. Major applications of the technology; herbicide, insect pest, disease (bacterial, fungal and viral) resistance; stress tolerance; and crop improvement are discussed in detail, together with the concepts and application of molecular 'pharming'. Finally, the text summarises the current and future prospects of GM plants.

The text focuses on the application of the genetic manipulation of plants, and while not within the scope of this text, 'molecular breeding' is another area that can be considered plant biotechnology; that is the use of molecular biology techniques to speed up the screening in conventional breeding programmes. Molecular breeding is widely used, and does not face the controversy seen with genetically manipulated plants.
Each chapter is well structured, containing line drawings that clearly and simply illustrate concepts in the text. Text boxes dispersed throughout the text are used to summarise particular topics, for example 'plastid transformation' and 'The Pusztai affair' (effects of feeding GM potatoes to laboratory animals). Appended to each chapter is a further reading list directing the reader to key general and specific papers and internet sites. The text itself has a companion web site, www.oup.com/uk/best.textbooks/biochemistry/slater, which contains downloadable figures, links to relevant websites that include additional information and discussions, news and updates about the field of plant biotechnology including updates of issues raised in the text, and case-study problems aimed at students. The concept of a related web site is very encouraging, and it is hoped that the promised updates, etc. will occur and in a timely manner.

The first two sections of the text contain information that would be expected of any book in this field. They are up to date, and the selection of examples of genetic modifications is well chosen. What makes this text complete is the final chapter where the authors examine some of the broader issues surrounding, and future applications of the technology (many of these applications are already in development), having explained the technology in preceding chapters. The major concerns surrounding adaptation of the technology are introduced. These topics warrant much more detailed discussion; however, this is beyond the scope of the text, the companion web site directing the reader to the appropriate resources. Also included is an introduction to EU legislation regarding the release of GM plants within Europe, which until recently has had an effective moratorium on release. Recently, however (and since publication of this text), legislation has changed, and in the UK the environmental release agency ACRE concluded that '....if 'herbicide resistant maize' were to be grown and managed as in the farm-scale evaluations this would not result in adverse effects, as defined and assessed by criteria specified in Directive 2001/18/EC, compared with conventionally managed maize'. Thus plant technology may indeed be entering a new phase in its history.

RS Conlan

School of Biological Sciences, University of Wales Swansea, Singleton Park, Swansea SA2 8PP, UK E-mail: r.s.conlan@swan.ac.uk 